-
$=$
$=$
-
-
-
-
-
-
-
-

$-$

Division of Social
Medicine and Public
Health, Peking Union
Medical College,
Beijing, People's
Republic of China
SQ Wang
SQ W He

-. National Institutes of Health, Bethesda, Maryland, USA JJ Yu

Battelle Memorial Institute Atlanta

Operation, Atlanta,

Georgia, USA

BP Zhu

$\sim$

Department of

Internal Medicine,

University of

Michigan Medical

Center, Ann Arbor,

Michigan, USA

$M$ Liu

Correspondence to: Bao-

Ping Zhu, Battelle Memoria

Institute, $\mathrm{c} / \mathrm{o}$ Office on

Smoking and Health,

National Center for Chronic

Disease Prevention and

Health Promotion, Centers

for Disease Control and

Prevention, MailStop K-50,

1600 Clifton Road NE,

Atlanta, Georgia 30333,

USA.

\title{
Cigarette smoking and its risk factors among senior high school students in Beijing, China, $1988^{\star}$
}

\author{
Shu-Qi Wang, Jing-Jie Yu, Bao-Ping Zhu, Ming Liu, Guan-Qing He
}

\begin{abstract}
Objective - To study the prevalence and behavioural patterns of, and risk factors for, cigarette smoking among senior high school students in Beijing, China.

Design - A sample of 3823 senior high school students (primarily ages 16-19 years) in 78 classes were selected from 30 schools in Beijing in 1988, utilising a multi-stage stratified cluster sampling scheme.
\end{abstract}

Setting - Students in a selected class met in a classroom to fill out a selfadministered anonymous questionnaire, under the supervision of trained interviewers.

Results - Smoking at least occasionally was reported by $46.0 \%$ of the males and $5.5 \%$ of the females, although daily cigarette consumption was low. Smoking prevalence increased with age among males. About $2 \%$ of the ever-smokers reported trying their first cigarette before the age of five years. The most frequently cited reasons for initiation were "elegance", "curiosity", and "imitation". The most important reasons for frequent smoking were "elegance", "fashion", "dispelling melancholy", and "seeking stimulation". Inhaling was practised by $40.2 \%$ of the male and $13.8 \%$ of the female daily or weekly smokers. Eighty-six percent of the males and $69 \%$ of the females usually smoked with friends or schoolmates. While $16.6 \%$ of the males often smoked at home, no females did so. Smoking was related to a combination of psychological, familial, and socio-school factors, with peer pressure being the strongest influence.

Conclusions-Risk factors for smoking among Chinese students are similar to those of industrialised countries. Efforts to monitor prevalence and risk factors of smoking among Chinese adolescents are recommended.

(Tobacco Control 1994; 3: 107-114)

\section{Introduction}

Smoking presents a serious health threat in China. National survey data showed that in

* This paper is based on a portion of Dr Zhu's $\mathrm{PhD}$ dissertation.
$1984,61 \%$ of males and $7 \%$ of females in China over the age of 15 smoked, ${ }^{1,2}$ representing 250-300 million smokers, or approximately $25 \%$ of all the smokers in the world. ${ }^{2,3}$

Researchers in western countries have stressed the importance of studying smoking among adolescents since most smokers start smoking during adolescence or early adulthood ${ }^{4,5}$ and those with a younger age of initiation are more likely to become regular smokers. ${ }^{5}$ In addition, smokers who start smoking earlier will experience higher mortality from smoking-related diseases. ${ }^{6-8}$ However, there have been few studies on smoking among adolescents in China. In order to identify the prevalence and behavioural patterns of, and risk factors for, cigarette smoking among Chinese students, we employed a school-based self-administered questionnaire among students at all school levels in Beijing during 1988. This paper reports findings for the senior high school students.

\section{Subjects and methods}

TARGET POPULATION

Government statistics $\nmid$ show that in 1988, $99.7 \%$ of school-age children (age seven) in Beijing entered elementary schools; $98.5 \%$ of elementary school graduates entered junior high schools; and $63.2 \%$ of junior high school graduates continued on to senior high schools. A majority of the children started senior high school at age 16 and graduated at 19. A small number of students entered senior high schools earlier than 16 years old because they began school earlier or had skipped grades due to their outstanding performance. On the other hand, some students stayed in senior high schools until later than 19 years old because they started elementary school later or had to repeat one or more grades. During 1988, there were 108858 senior high school students (55012 males and 53846 females) in 433 senior high schools. All senior high school graduates are required to take the annual National College Entrance Examination in order to be enrolled in universities, colleges, or vocational schools. In $1988,44.2 \%$ of the graduates in Beijing passed the examination and thus were able to continue higher education, while those

$\dagger$ Educational statistics of Beijing, school years 1980-1990. Beijing Governor's Office on Culture and Education, April 1991 (in Chinese). 
who failed would either start to work or stay in senior high school to retake the exam. The latter were excluded from this study because they are not listed on the school rosters and it was difficult to obtain their compliance.

\section{SAMPLING PROCEDURE}

A two-stage stratified cluster sampling method was used. In the first stage, the 433 schools were classified into two strata: urban schools (ie, those located in the city proper and the county towns) and rural schools (ie, those in the outlying areas of the counties). Schools were chosen randomly from each stratum. At the second stage, classes were selected randomly from the schools. Once a class was selected, all students in the class were surveyed. The sampling process was similar to that of the junior high school students described in more detail elsewhere. ${ }^{9}$ In total, 78 classes from 30 senior high schools were selected, and the total number of students surveyed was 3972 .

\section{SAMPLE SIZE}

A previous survey involving two classes in a senior high school estimated that the smoking prevalence was $12 \%$ (Beijing Education $\mathrm{Bu}-$ reau 1987, personal communication). Assuming the non-participation rate to be less than $15 \%$, and using the formula provided by Snedecor and William, ${ }^{10}$ the required sample size was calculated to be approximately 3500 . The sampling process generated 3972 students (as mentioned above), from which 149 were excluded because of absence during the survey or incorrect completion of the questionnaires. The effective sample size was 3823 (1923 males and 1900 females), yielding a response rate of $96.3 \%$. The gender ratio (males to females) in this sample (1.01) was similar to that of the source population (1.02). The age distribution of the sample was as follows: < 16 years: 37 $(1 \%) ; 16$ years: $403(11 \%) ; 17$ years: 1347 $(35 \%) ; 18$ years: $1374(36 \%) ; 19$ years : 508 $(13 \%) ; 20$ years or older: $154(4 \%)$.

\section{SURVEY METHOD}

Students were surveyed in the classroom where they usually had classes. They were informed by trained interviewers that the survey was anonymous and that individual responses would not be disclosed to their teachers or parents. This fact was strongly emphasised in order to reduce the possibility of underreporting by smokers.

\section{QUESTIONNAIRE}

Information in the following five areas was collected: (a) social and demographic items; (b) smoking status; (c) age of and reason for initiation; (d) behavioural patterns of smoking, (ie, frequency, daily consumption, inhalation practice, reasons for, settings and companions of smoking); and (e) risk factors for smoking, including psychological, familial, and socio- school variables. The questionnaire, translated into English is reproduced in the Appendix.

\section{DEFINITION OF SMOKING}

Since tobacco products other than cigarettes are practically unknown among Beijing students, only cigarette smoking was considered in this study. Daily smokers were those who smoked every day (or almost every day); weekly smokers were those who smoked at least once a week, but not daily; occasional smokers included those who smoked less often than once a week, only on special occasions, and those who had puffed a few times; neversmokers were those who had never tried a cigarette. Because the numbers of daily and weekly smokers were small, they were combined to stabilise some of the estimates during data analysis, and are referred to as frequent smokers in this paper for convenience. Inhaling smokers were those who often inhaled; mouth smokers were those who took smoke in their mouths and puffed out immediately. Exclusive categories were given for reasons and settings for smoking (ie, only one answer could be given to each item).

\section{STATISTICAL ANALYSIS}

$\mathrm{BMDP}^{11}$ and SAS ${ }^{12}$ were used for data analysis. Stepwise logistic regression analysis ${ }^{11,14}$ was performed on a sex-specific basis to evaluate risk factors for smoking. Before performing logistic regression analysis, principal component analysis ${ }^{12,13}$ was applied to psychological, familial, and socio-school variables separately to generate a number of factors. From each factor, the variable with the largest factor loading was selected for the stepwise fitting in the logistic model. The HosmerLemeshow statistic ${ }^{11,14}$ was used to assess the goodness-of-fit of the models. In fitting the logistic models, occasional smokers were excluded because, by definition, this category covered a wide range, from those who smoked less often than once a week to those who might have had only a few puffs. Approximately $2 \%$ of the records had missing values for some of the variables. Instead of deleting these records, we replaced the missing values with the modes of the corresponding variables during the analysis.

\section{Results}

PREVALENCE

Of the 3823 students (1923 males and 1900 females) in the sample, $25.9 \%(46.0 \%$ of males and $5.5 \%$ of females) had smoked cigarettes (ie, at least a few puffs). The proportions of daily, weekly, and occasional smokers were $8.8 \%, 9.9 \%$ and $27.2 \%$ among males, and $0.6 \%, 0.9 \%$, and $4.0 \%$ among females, respectively. Figures 1 and 2 present smoking prevalence by age for males and females, respectively. Smoking was much more prevalent among males than females. In males, smoking rates increased with age. In females, due to the small number of smokers observed, the relationship between smoking prevalence and age was unclear. 


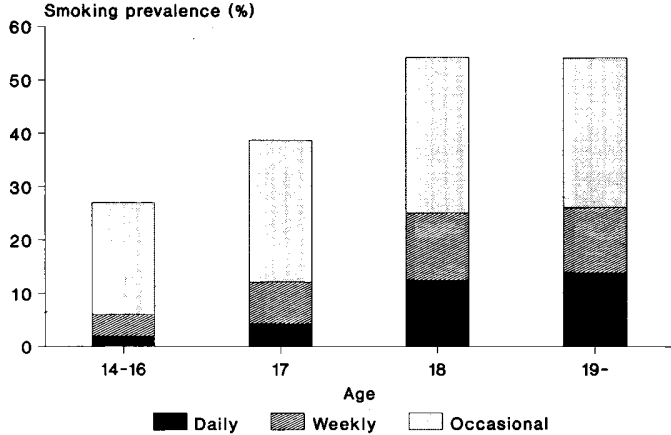

Figure 1 Smoking prevalence among male senior high school students in Beijing, 1988.

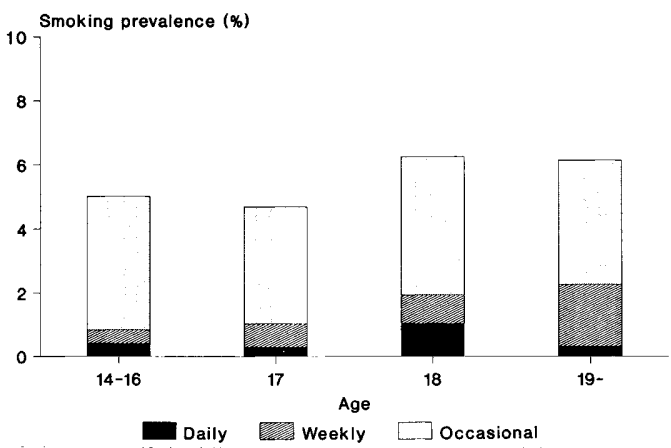

Figure 2 Smoking prevalence among female senior high school students in Beijing, 1988.

Table 1 Age of and reason for trying the first cigarette by ever-smokers. Senior high school students, Beijing, 1988

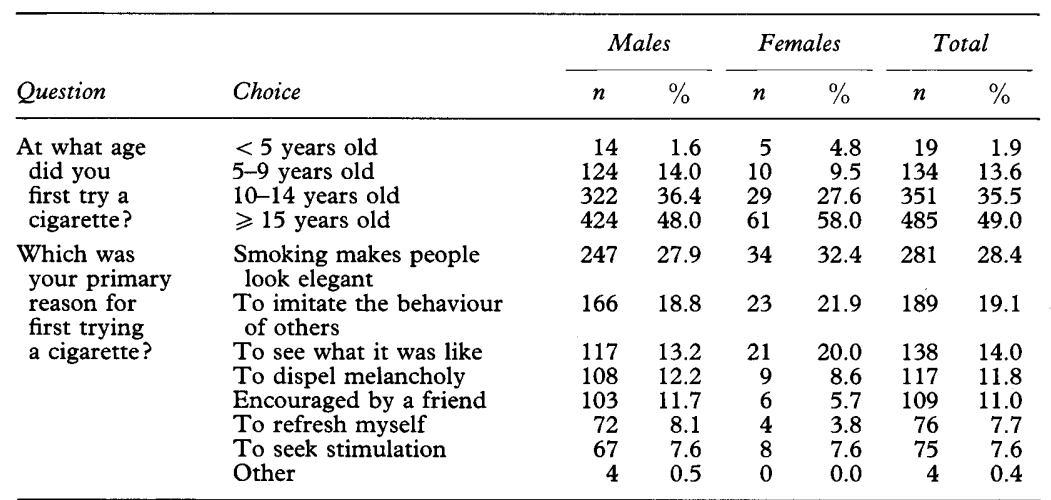

Table 2 Behavioural patterns of smoking among frequent smokers. Senior high school students, Beijing, 1988

\begin{tabular}{|c|c|c|c|c|c|c|c|c|}
\hline 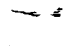 & & & & & & ales & & tal \\
\hline 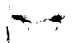 & Question & Choice & $n$ & $\%$ & $n$ & $\%$ & $n$ & $\%$ \\
\hline- & $\begin{array}{l}\text { On average, how } \\
\text { many cigarettes } \\
\text { do you smoke per } \\
\text { day? }\end{array}$ & $\begin{array}{l}<5 \\
5-9 \\
\geqslant 10\end{array}$ & $\begin{array}{r}338 \\
17 \\
6\end{array}$ & $\begin{array}{r}93.6 \\
4.7 \\
1.7\end{array}$ & $\begin{array}{r}28 \\
1 \\
0\end{array}$ & $\begin{array}{r}96.6 \\
3.4 \\
0.0\end{array}$ & $\begin{array}{r}366 \\
18 \\
6\end{array}$ & $\begin{array}{r}93.9 \\
4.6 \\
1.5\end{array}$ \\
\hline$\cdots$ & $\begin{array}{l}\text { When you smoke, } \\
\text { do you usually } \\
\text { inhale or not? }\end{array}$ & $\begin{array}{l}\text { Inhale } \\
\text { Just take the smoke } \\
\text { in my mouth }\end{array}$ & $\begin{array}{r}145 \\
61\end{array}$ & $\begin{array}{l}40.2 \\
16.9\end{array}$ & $\begin{array}{l}4 \\
8\end{array}$ & $\begin{array}{l}13.8 \\
27.6\end{array}$ & $\begin{array}{r}149 \\
69\end{array}$ & $\begin{array}{l}38.2 \\
17.7\end{array}$ \\
\hline$=\ldots$ & & $\begin{array}{l}\text { Both, can't say which } \\
\text { is more often }\end{array}$ & 155 & 42.9 & 17 & 58.6 & 172 & 44.1 \\
\hline+2 & $\begin{array}{l}\text { Which is your } \\
\text { primary reason }\end{array}$ & $\begin{array}{l}\text { Smoking makes people } \\
\text { look elegant }\end{array}$ & 159 & 44.0 & 2 & 6.9 & 161 & 41.3 \\
\hline$-\cdots$ & for smoking now? & $\begin{array}{l}\text { To be fashionable } \\
\text { To dispel melancholy } \\
\text { To seek stimulation } \\
\text { To adapt to the custom } \\
\text { To refresh myself } \\
\text { Other }\end{array}$ & $\begin{array}{l}36 \\
20 \\
39 \\
32 \\
24 \\
51\end{array}$ & $\begin{array}{r}10.0 \\
5.5 \\
10.8 \\
8.9 \\
6.7 \\
14.1\end{array}$ & $\begin{array}{l}8 \\
7 \\
8 \\
0 \\
0 \\
4\end{array}$ & $\begin{array}{r}27.6 \\
24.1 \\
27.6 \\
0.0 \\
0.0 \\
0.0\end{array}$ & $\begin{array}{l}44 \\
27 \\
47 \\
32 \\
24 \\
55\end{array}$ & $\begin{array}{r}11.3 \\
6.9 \\
12.1 \\
8.2 \\
6.2 \\
14.1\end{array}$ \\
\hline$-x$ & $\begin{array}{l}\text { Where do you } \\
\text { usually smoke? }\end{array}$ & $\begin{array}{l}\text { My home } \\
\text { Way to / from school } \\
\text { School } \\
\text { Friend's home } \\
\text { Other }\end{array}$ & $\begin{array}{r}60 \\
115 \\
39 \\
75 \\
72\end{array}$ & $\begin{array}{l}16.6 \\
31.9 \\
10.8 \\
20.8 \\
19.9\end{array}$ & $\begin{array}{r}0 \\
14 \\
3 \\
10 \\
2\end{array}$ & $\begin{array}{r}0.0 \\
48.3 \\
10.3 \\
34.5 \\
6.9\end{array}$ & $\begin{array}{r}60 \\
129 \\
42 \\
85 \\
74\end{array}$ & $\begin{array}{l}15.4 \\
33.1 \\
10.8 \\
21.8 \\
19.0\end{array}$ \\
\hline- & $\begin{array}{l}\text { Whom do you } \\
\text { usually smoke } \\
\text { with? }\end{array}$ & $\begin{array}{l}\text { I usually smoke alone } \\
\text { Friends or schoolmates } \\
\text { Family members } \\
\text { Other }\end{array}$ & $\begin{array}{r}16 \\
309 \\
4 \\
32\end{array}$ & $\begin{array}{r}4.4 \\
85.6 \\
1.1 \\
8.9\end{array}$ & $\begin{array}{r}4 \\
20 \\
0 \\
5\end{array}$ & $\begin{array}{r}13.8 \\
69.0 \\
0.0 \\
17.2\end{array}$ & $\begin{array}{r}20 \\
329 \\
4 \\
37\end{array}$ & $\begin{array}{r}5.1 \\
84.4 \\
1.0 \\
9.5\end{array}$ \\
\hline
\end{tabular}

INITIATION

To all students who reported ever smoking a cigarette (ie, daily, weekly, or occasional smokers), we asked questions about their age of and reasons for first trying a cigarette. Table 1 summarises the responses. About $2 \%$ of the ever-smokers reported first trying a cigarette before age five. For both males and females, the most frequently cited reasons for first trying a cigarette were "elegance" ("believed smoking makes people look elegant"), "curiosity" ("to see what it was like"), and "imitation" ("to imitate the behaviour of others").

\section{BEHAVIOURAL PATTERNS}

Five questions were asked of the daily and weekly smokers about their behavioural patterns of smoking. As noted above, we combined data for the daily and weekly smokers because the numbers in both categories are small. For convenience, we will refer to this combined category as "frequent smokers".

Table 2 shows the behavioural patterns of the 390 frequent smokers (361 males and 29 females). Most of them were light smokers (ie, $<$ five cigarettes a day). More males than females smoked $\geqslant$ five cigarettes per day $(6.4 \%$ versus $3.4 \%)$, but this difference was not statistically significant $(\mathrm{p}>0.05)$. A significantly higher proportion of male frequent smokers inhaled compared to females $(40.2 \%$ versus $13.8 \%, \mathrm{p}<0.01)$. While "elegance" was the most important reason for smoking by males, females tended to smoke "to be fashionable", "to dispel melancholy", and "to seek stimulation". For both males and females, on the "way to / from school" was the main setting for their smoking. Many males smoked at home $(16.6 \%)$, but no females did so. Most smokers usually smoked with their friends or schoolmates; few of them tended to smoke alone.

\section{RISK FACTORS}

Three categories of risk factors for smoking were investigated: psychological, familial, and socio-school. The results are shown in tables 3, 4 , and 5. To summarise, for psychological variables, smoking was more likely among students with negative attitudes toward attending school " for the sake of parents" or "for a diploma") and goals while attending school ("enjoy life" or to "be able to graduate and get a diploma"), unwillingness to respect discipline, and disagreement with the relationship between smoking and health. On the other hand, students who were planning higher education after graduation were less likely to smoke. Students' pessimism about life was only weakly correlated with smoking, albeit statistically significant (Spearman correlation coefficient $=0.018, \mathrm{p}=0.016$ ) .

For familial variables, parents' low education and occupation status, and smoking among parents and siblings were positively associated with smoking among students. The more parents asked their children to buy 
Table 3 Psychological variables and smoking. Senior high school students, Beijing, 1988

\begin{tabular}{|c|c|c|c|c|c|c|}
\hline \multirow[b]{2}{*}{ Variable } & \multirow[b]{2}{*}{ Category } & \multicolumn{4}{|c|}{ Smoking status } & \multirow[b]{2}{*}{$n$} \\
\hline & & Daily & Weekly & Occasional & Never & \\
\hline \multirow[t]{2}{*}{$\begin{array}{l}\text { Motive for } \\
\text { attending } \\
\text { school }\end{array}$} & $\begin{array}{l}\text { For my personal interests } \\
\text { To better serve the public } \\
\text { To help me identify my personal values } \\
\text { and ideas }\end{array}$ & $\begin{array}{l}3.4 \\
3.5 \\
4.5\end{array}$ & $\begin{array}{l}5.0 \\
6.0 \\
5.7\end{array}$ & $\begin{array}{l}14.0 \\
14.8 \\
15.3\end{array}$ & $\begin{array}{l}77.6 \\
75.7 \\
74.5\end{array}$ & $\begin{array}{r}955 \\
519 \\
1218\end{array}$ \\
\hline & $\begin{array}{l}\text { For the sake of my parents } \\
\text { For a diploma } \\
\text { Other }\end{array}$ & $\begin{array}{l}5.8 \\
9.5 \\
6.0\end{array}$ & $\begin{array}{l}4.9 \\
8.3 \\
4.3\end{array}$ & $\begin{array}{l}19.0 \\
16.6 \\
17.6\end{array}$ & $\begin{array}{l}70.3 \\
65.6 \\
72.2\end{array}$ & $\begin{array}{l}411 \\
253 \\
467\end{array}$ \\
\hline $\begin{array}{l}\text { Goals while } \\
\text { attending } \\
\text { school }\end{array}$ & $\begin{array}{l}\text { Achieve all-around success } \\
\text { Achieve good scores } \\
\text { Enjoy life } \\
\text { Be able to graduate and get a diploma } \\
\text { Other }\end{array}$ & $\begin{array}{r}2.0 \\
3.3 \\
7.0 \\
19.7 \\
4.7\end{array}$ & $\begin{array}{r}3.6 \\
7.0 \\
6.9 \\
11.7 \\
5.1\end{array}$ & $\begin{array}{l}13.3 \\
15.9 \\
17.9 \\
18.4 \\
17.7\end{array}$ & $\begin{array}{l}81.1 \\
73.8 \\
68.2 \\
50.2 \\
72.5\end{array}$ & $\begin{array}{r}1610 \\
511 \\
738 \\
223 \\
741\end{array}$ \\
\hline $\begin{array}{l}\text { Goals after } \\
\text { graduation }\end{array}$ & $\begin{array}{l}\text { Pursue higher education } \\
\text { Find a good job } \\
\text { Make money } \\
\text { Other }\end{array}$ & $\begin{array}{r}1.9 \\
7.0 \\
17.0 \\
4.9\end{array}$ & $\begin{array}{r}4.1 \\
7.0 \\
11.3 \\
4.6\end{array}$ & $\begin{array}{l}15.4 \\
15.1 \\
20.0 \\
15.8\end{array}$ & $\begin{array}{l}78.6 \\
70.8 \\
51.7 \\
74.7\end{array}$ & $\begin{array}{r}2067 \\
1080 \\
265 \\
411\end{array}$ \\
\hline $\begin{array}{l}\text { Attitude } \\
\text { toward } \\
\text { life }\end{array}$ & $\begin{array}{l}\text { Optimistic } \\
\text { In-between } \\
\text { Pessimistic }\end{array}$ & $\begin{array}{l}4.3 \\
5.0 \\
5.2\end{array}$ & $\begin{array}{l}5.4 \\
5.3 \\
5.6\end{array}$ & $\begin{array}{l}15.6 \\
14.4 \\
16.3\end{array}$ & $\begin{array}{l}74.6 \\
75.4 \\
72.8\end{array}$ & $\begin{array}{r}1958 \\
585 \\
1280\end{array}$ \\
\hline $\begin{array}{l}\text { Attitude } \\
\text { toward } \\
\text { respecting } \\
\text { discipline }\end{array}$ & $\begin{array}{l}\text { Willing to respect discipline } \\
\text { Follow other people's suit } \\
\text { Unwilling to respect discipline }\end{array}$ & $\begin{array}{l}3.0 \\
5.8 \\
8.0\end{array}$ & $\begin{array}{l}4.7 \\
6.0 \\
7.0\end{array}$ & $\begin{array}{l}12.7 \\
17.6 \\
21.1\end{array}$ & $\begin{array}{l}79.6 \\
70.7 \\
63.9\end{array}$ & $\begin{array}{r}2015 \\
1108 \\
700\end{array}$ \\
\hline $\begin{array}{l}\text { Beliefs on } \\
\text { smoking and } \\
\text { health }\end{array}$ & $\begin{array}{l}\text { Believe that smoking harms health } \\
\text { Have no idea } \\
\text { Disagree that smoking harms health }\end{array}$ & $\begin{array}{r}4.1 \\
6.6 \\
18.3\end{array}$ & $\begin{array}{l}5.4 \\
4.4 \\
9.6\end{array}$ & $\begin{array}{l}15.3 \\
15.8 \\
25.2\end{array}$ & $\begin{array}{l}75.1 \\
73.2 \\
47.0\end{array}$ & $\begin{array}{r}3436 \\
272 \\
115\end{array}$ \\
\hline
\end{tabular}

* Spearman correlation coefficient $=0.018, \mathrm{p}=0.016$

Table 4 Familial variables and smoking. Senior high school students, Beijing, 1988

\begin{tabular}{|c|c|c|c|c|c|c|}
\hline \multirow[b]{2}{*}{ Variable } & \multirow[b]{2}{*}{ Category } & \multicolumn{4}{|c|}{ Smoking status } & \multirow[b]{2}{*}{$n$} \\
\hline & & Daily & Weekly & Occasional & Never & \\
\hline $\begin{array}{l}\text { Father's } \\
\text { education level }\end{array}$ & $\begin{array}{l}\text { University/college } \\
\text { High school } \\
\text { Elementary school } \\
\text { No education }\end{array}$ & $\begin{array}{l}2.9 \\
5.5 \\
7.8 \\
6.7\end{array}$ & $\begin{array}{l}5.1 \\
5.3 \\
7.1 \\
6.7\end{array}$ & $\begin{array}{l}15.9 \\
15.9 \\
14.2 \\
14.8\end{array}$ & $\begin{array}{l}76.1 \\
73.3 \\
71.0 \\
71.9\end{array}$ & $\begin{array}{r}1551 \\
1713 \\
424 \\
135\end{array}$ \\
\hline $\begin{array}{l}\text { Mother's } \\
\text { education level }\end{array}$ & $\begin{array}{l}\text { University/college } \\
\text { High school } \\
\text { Elementary school } \\
\text { No education }\end{array}$ & $\begin{array}{l}2.9 \\
5.0 \\
7.1 \\
3.6\end{array}$ & $\begin{array}{l}5.3 \\
5.1 \\
5.7 \\
8.4\end{array}$ & $\begin{array}{l}14.6 \\
15.7 \\
16.2 \\
17.9\end{array}$ & $\begin{array}{l}77.2 \\
74.2 \\
71.0 \\
70.1\end{array}$ & $\begin{array}{r}924 \\
2045 \\
580 \\
274\end{array}$ \\
\hline $\begin{array}{l}\text { Father's } \\
\text { occupation }\end{array}$ & $\begin{array}{l}\text { Scientist, teacher, } \\
\text { doctor }\end{array}$ & 3.3 & 4.7 & 15.7 & 76.3 & 892 \\
\hline & $\begin{array}{l}\text { Gov't official } \\
\text { Worker } \\
\text { Farmer } \\
\text { Other }\end{array}$ & $\begin{array}{l}3.5 \\
7.0 \\
7.2 \\
6.2\end{array}$ & $\begin{array}{l}4.9 \\
6.6 \\
7.4 \\
4.5\end{array}$ & $\begin{array}{l}16.2 \\
13.7 \\
18.3 \\
14.9\end{array}$ & $\begin{array}{l}75.4 \\
72.8 \\
67.2 \\
74.4\end{array}$ & $\begin{array}{r}1479 \\
805 \\
405 \\
242\end{array}$ \\
\hline $\begin{array}{l}\text { Mother's } \\
\text { occupation }\end{array}$ & $\begin{array}{l}\text { Scientist, teacher, } \\
\text { doctor }\end{array}$ & 3.2 & 5.0 & 16.6 & 75.2 & 1092 \\
\hline & $\begin{array}{l}\text { Gov't official } \\
\text { Worker } \\
\text { Farmer } \\
\text { Other }\end{array}$ & $\begin{array}{l}4.6 \\
5.1 \\
5.2 \\
6.9\end{array}$ & $\begin{array}{l}4.9 \\
5.4 \\
6.4 \\
6.3\end{array}$ & $\begin{array}{l}15.3 \\
12.7 \\
18.1 \\
17.8\end{array}$ & $\begin{array}{l}75.3 \\
76.9 \\
70.3 \\
69.0\end{array}$ & $\begin{array}{r}655 \\
1026 \\
498 \\
552\end{array}$ \\
\hline $\begin{array}{l}\text { Father's } \\
\text { smoking status }\end{array}$ & $\begin{array}{l}\text { Daily } \\
\text { Weekly } \\
\text { Occasional } \\
\text { Ex- } \\
\text { Never }\end{array}$ & $\begin{array}{l}6.3 \\
4.0 \\
2.7 \\
6.2 \\
3.3\end{array}$ & $\begin{array}{l}6.1 \\
4.6 \\
6.3 \\
5.0 \\
4.4\end{array}$ & $\begin{array}{l}16.9 \\
15.5 \\
15.3 \\
18.5 \\
13.0\end{array}$ & $\begin{array}{l}70.7 \\
76.0 \\
75.7 \\
70.3 \\
79.3\end{array}$ & $\begin{array}{r}1566 \\
329 \\
589 \\
340 \\
999\end{array}$ \\
\hline $\begin{array}{l}\text { Mother's } \\
\text { smoking status }\end{array}$ & $\begin{array}{l}\text { Daily } \\
\text { Weekly } \\
\text { Occasional } \\
\text { Ex- } \\
\text { Never }\end{array}$ & $\begin{array}{r}18.2 \\
7.1 \\
5.0 \\
11.1 \\
4.1\end{array}$ & $\begin{array}{r}5.0 \\
8.2 \\
4.5 \\
13.0 \\
5.4\end{array}$ & $\begin{array}{l}16.5 \\
18.8 \\
20.8 \\
11.1 \\
15.3\end{array}$ & $\begin{array}{l}60.3 \\
65.9 \\
69.8 \\
64.8 \\
75.2\end{array}$ & $\begin{array}{r}121 \\
85 \\
202 \\
54 \\
3361\end{array}$ \\
\hline $\begin{array}{l}\text { Parents' attitude } \\
\text { towards smoking }\end{array}$ & $\begin{array}{l}\text { Strongly against } \\
\text { Against } \\
\text { Not against } \\
\text { I don't know }\end{array}$ & $\begin{array}{r}2.0 \\
6.7 \\
15.7 \\
2.0\end{array}$ & $\begin{array}{r}3.3 \\
7.7 \\
10.2 \\
4.3\end{array}$ & $\begin{array}{l}14.4 \\
20.4 \\
15.7 \\
10.5\end{array}$ & $\begin{array}{l}80.3 \\
65.3 \\
58.4 \\
83.2\end{array}$ & $\begin{array}{r}1642 \\
1169 \\
363 \\
649\end{array}$ \\
\hline $\begin{array}{l}\text { Smoking among } \\
\text { siblings }\end{array}$ & $\begin{array}{l}\text { No } \\
\text { At least one }\end{array}$ & $\begin{array}{l}3.9 \\
7.3\end{array}$ & $\begin{array}{l}4.9 \\
7.2\end{array}$ & $\begin{array}{l}15.2 \\
17.0\end{array}$ & $\begin{array}{l}75.9 \\
68.5\end{array}$ & $\begin{array}{r}2905 \\
918\end{array}$ \\
\hline $\begin{array}{l}\text { Buying cigarettes } \\
\text { for parents }\end{array}$ & $\begin{array}{l}\text { Never } \\
\text { Occasionally } \\
\text { Often }\end{array}$ & $\begin{array}{r}3.5 \\
5.4 \\
18.2\end{array}$ & $\begin{array}{l}4.6 \\
6.7 \\
6.8\end{array}$ & $\begin{array}{l}13.0 \\
19.4 \\
21.2\end{array}$ & $\begin{array}{l}78.8 \\
68.4 \\
53.8\end{array}$ & $\begin{array}{r}2292 \\
1399 \\
132\end{array}$ \\
\hline
\end{tabular}

cigarettes, the more likely the children would be to smoke. Conversely, if parents were against smoking, their children would be less likely to smoke. (Smoking prevalence was also low among those whose parents' attitude was unknown, but we believe that this is because many of these students did not smoke in the first place.) A positive correlation existed between children and their parents with respect to the frequency of current smoking. However, it was somewhat unexpected that smoking among students whose parents were ex-smokers (who used to smoke frequently but had quit smoking for at least a month) was as prevalent as among those whose parents were frequent current smokers. For socio-school 
Table 5 Social-school variables and smoking. Senior high school students, Beijing, $\div \quad 1988$

\begin{tabular}{|c|c|c|c|c|c|c|c|}
\hline \multirow{2}{*}{$\Rightarrow$} & \multirow[b]{2}{*}{ Variable } & \multirow[b]{2}{*}{ Category } & \multicolumn{4}{|c|}{ Smoking status } & \multirow[b]{2}{*}{$n$} \\
\hline & & & Daily & Weekly & Occasional & Never & \\
\hline & $\begin{array}{l}\text { School } \\
\text { performance }\end{array}$ & $\begin{array}{l}\text { A-level } \\
\text { B-level } \\
\text { C-level }\end{array}$ & $\begin{array}{l}2.6 \\
4.6 \\
8.7\end{array}$ & $\begin{array}{l}4.2 \\
5.3 \\
8.0\end{array}$ & $\begin{array}{l}13.9 \\
15.2 \\
20.5\end{array}$ & $\begin{array}{l}79.3 \\
74.9 \\
62.9\end{array}$ & $\begin{array}{r}861 \\
2396 \\
566\end{array}$ \\
\hline & $\begin{array}{l}\text { Smoking among } \\
\text { teachers }\end{array}$ & $\begin{array}{l}\text { No } \\
\text { At least one }\end{array}$ & $\begin{array}{l}2.4 \\
5.4\end{array}$ & $\begin{array}{l}3.8 \\
5.9\end{array}$ & $\begin{array}{l}12.5 \\
16.5\end{array}$ & $\begin{array}{l}81.2 \\
72.2\end{array}$ & $\begin{array}{r}821 \\
3002\end{array}$ \\
\hline & $\begin{array}{l}\text { Smoking among } \\
\text { close friends }\end{array}$ & $\begin{array}{l}\text { No } \\
\text { At least one }\end{array}$ & $\begin{array}{l}0.4 \\
9.6\end{array}$ & $\begin{array}{r}1.0 \\
10.5\end{array}$ & $\begin{array}{r}6.1 \\
26.5\end{array}$ & $\begin{array}{l}92.5 \\
53.3\end{array}$ & $\begin{array}{l}2029 \\
1794\end{array}$ \\
\hline
\end{tabular}

$-\infty$

variables, smoking of students was related to poor school performance, and having teachers and close friends who smoked.

Of all the three categories of variables investigated, "having at least one close friend who smoked" was the strongest correlate of smoking. The prevalence of daily, weekly, and occasional smoking among those with at least one smoking friend was $24.0,10.5$, and 4.3 times higher than those without smoking friends, respectively.

Table 6 presents the results of the logistic regression analysis. The principle component analysis generated 10 factors. From these factors, 10 variables were chosen, along with age, for the backward stepwise fit of the logistic models for males and females separately. The Hosmer-Lemeshow test suggests that both models fit well.

$\mathrm{OR}=$ Odds Ratio $; \mathrm{CI}=$ Confidence Interval

$\star$ Reference group; (-) Variable not entered into the model
All variables but "mother's education level" entered the model for the males. After adjusting for the other variable, "smoking among close friends" (having at least one close friend who smoked, odds ratio $(O R)=16.50$ ) was the strongest correlate of smoking. Older age, "beliefs on smoking and health" (disagreeing that smoking harms health), "parents' attitude toward smoking" (not against), and "intention after graduation" (make money / find a good job) were also strongly associated with smoking.

Only four variables (ie, "parents' attitude toward smoking", "buying cigarettes for parents", "goals after graduation", and "smoking among close friends") entered the model for females, most probably because there are only 29 observations with outcome variable equal to 1 ("frequent smokers" ie, daily or weekly smokers) in the sample. The ORs associated with these variables are all large (most of them greater than 2.5), but because of the small number of "success" observations, the confidence intervals are also wide.

\section{Discussion}

We did not use biological markers to validate the smoking status reported by the students because this would have enormously increased the cost of the research, given the large sample size. In addition, most of the practical bio-

Table 6 Logistic regression analysis of smoking risk factors. Senior high school students, Beijing, 1988

\begin{tabular}{|c|c|c|c|c|c|c|}
\hline \multirow[b]{2}{*}{ Variable } & \multicolumn{3}{|c|}{ Male } & \multicolumn{3}{|c|}{ Female } \\
\hline & Coefficient & $O R$ & $95 \% C I$ & Coefficient & $O R$ & $95 \% C I$ \\
\hline $\begin{array}{l}\text { Constant } \\
\text { Age }\end{array}$ & -6.5010 & & & -5.5790 & & \\
\hline $\begin{array}{l}\geqslant 19 \\
18 \\
17 \\
14-16\end{array}$ & $\begin{array}{l}1.4870 \\
1.6450 \\
1.0730\end{array}$ & $\begin{array}{l}4.42 \\
5.18 \\
2.92 \\
1.00^{\star}\end{array}$ & $\begin{array}{l}2.10-9.31 \\
2.53-10.60 \\
1.40-6.10\end{array}$ & & $(-)$ & $(-)$ \\
\hline $\begin{array}{l}\text { Mother's education level } \\
\text { Elementary school or less } \\
\text { High school } \\
\text { University/college }\end{array}$ & $(-)$ & $(-)$ & $(-)$ & $(-)$ & $(-)$ & $(-)$ \\
\hline $\begin{array}{l}\text { Smoking among siblings } \\
\text { At least one } \\
\text { No }\end{array}$ & 0.1734 & $\begin{array}{l}1.19 \\
1.00^{\star}\end{array}$ & $0.85-1.66$ & $(-)$ & $(-)$ & $(-)$ \\
\hline Parents' attitude toward smoking & & & & & & \\
\hline $\begin{array}{l}\text { Not against } \\
\text { Otherwise }\end{array}$ & 1.2030 & $\begin{array}{l}3.33 \\
1.00^{\star}\end{array}$ & $2.15-5.15$ & 0.9927 & $\begin{array}{l}2.70 \\
1.00^{\star}\end{array}$ & $1.10-6.63$ \\
\hline $\begin{array}{l}\text { Frequently } \\
\text { Occasionally } \\
\text { Never }\end{array}$ & $\begin{array}{l}0.6155 \\
0.1265\end{array}$ & $\begin{array}{l}1.85 \\
1.13 \\
1.00^{\star}\end{array}$ & $\begin{array}{l}0.94-3.65 \\
0.83-1.55\end{array}$ & $\begin{array}{l}1.2850 \\
0.2833\end{array}$ & $\begin{array}{l}3.82 \\
1.34 \\
1.00^{\star}\end{array}$ & $\begin{array}{l}0.76-17.30 \\
0.60-2.92\end{array}$ \\
\hline $\begin{array}{l}\text { Goal after graduation } \\
\text { Make money } \\
\text { Find a good job } \\
\text { Other } \\
\text { Pursue higher education }\end{array}$ & $\begin{array}{l}1.0740 \\
0.9121 \\
0.2849\end{array}$ & $\begin{array}{l}2.93 \\
2.49 \\
1.33 \\
1.00^{\star}\end{array}$ & $\begin{array}{l}1.83-4.68 \\
1.77-3.51 \\
0.80-2.20\end{array}$ & $\begin{array}{l}1.7410 \\
1.0870 \\
0.7908\end{array}$ & $\begin{array}{l}5.17 \\
2.94 \\
2.15 \\
1.00^{\star}\end{array}$ & $\begin{array}{l}1.64-19.80 \\
1.23-7.17 \\
0.57-8.51\end{array}$ \\
\hline $\begin{array}{l}\text { Attitude toward respecting discipline } \\
\text { Unwilling to respect discipline } \\
\text { Follow other people's suit } \\
\text { Willing to respect discipline }\end{array}$ & $\begin{array}{l}0.5213 \\
0.4613\end{array}$ & $\begin{array}{l}1.68 \\
1.59 \\
1.00^{\star}\end{array}$ & $\begin{array}{l}1.15-2.46 \\
1.13-2.23\end{array}$ & $(-)$ & $(-)$ & $(-)$ \\
\hline $\begin{array}{l}\text { Beliefs on smoking and health } \\
\text { Disagree that smoking harms health } \\
\text { Otherwise }\end{array}$ & 1.6030 & $\begin{array}{l}4.97 \\
1.00^{\star}\end{array}$ & $2.36-10.50$ & $(-)$ & $(-)$ & $(-)$ \\
\hline $\begin{array}{l}\text { School performance } \\
\text { C-level } \\
\text { B-level } \\
\text { A-level }\end{array}$ & $\begin{array}{l}0.5275 \\
0.3390\end{array}$ & $\begin{array}{l}1.69 \\
1.40 \\
1.00^{\star}\end{array}$ & $\begin{array}{l}1.03-2.78 \\
0.93-2.12\end{array}$ & $(-)$ & $(-)$ & $(-)$ \\
\hline $\begin{array}{l}\text { Smoking among close friends } \\
\text { At least one } \\
\text { No }\end{array}$ & 2.8020 & $\begin{array}{l}16.50 \\
1.00^{\star}\end{array}$ & $9.56-28.40$ & 1.2480 & $\begin{array}{l}3.37 \\
1.00^{\star}\end{array}$ & $1.64-7.37$ \\
\hline $\begin{array}{l}\text { Smoking among teachers } \\
\text { At least one } \\
\text { No }\end{array}$ & 0.6255 & $\begin{array}{l}1.87 \\
1.00^{\star}\end{array}$ & $1.23-2.84$ & $(-)$ & $(-)$ & $(-)$ \\
\hline
\end{tabular}

Goodness-of-fit test (Hosmer-Lemeshow): For the model of males: chi-square $=5.323, \mathrm{df}=8, \mathrm{p}=0.723$ 
logical markers are either insensitive or nonspecific in detecting very light and infrequent smoking. Instead, we made various efforts during the survey to minimise possible underreporting by the smokers, including using a self-administered anonymous questionnaire, asking the teachers to leave the classroom during the survey, and strongly emphasising the anonymity of questionnaire responses.

Another limitation of this study is that it did not include those who stayed in senior high school to retake the National College Entrance Examination, nor did it include high school dropouts. Thus the results may not be generalised to cover these two groups of youths. Studies in western countries have found that smoking prevalence among high school dropouts is much higher. ${ }^{4,5,15}$ As described above, the dropout rate among Chinese students is high. Studies on smoking among Chinese high school dropouts are needed.

The prevalence of daily smoking found in this study was much higher than that among high school students in the Beijing area found in the national survey $(8.84 \%$ vs $4.17 \%$ for males, and $0.58 \%$ vs $0.00 \%$ for females, respectively), ${ }^{3}$ although the age group covered was similar in the two surveys: 15 years old and above in the national survey, ${ }^{3}$ and 14 and above in this study (only six students in our sample were 14 years old). This discrepancy may be partly due to the fact that the national survey was conducted in 1984. Also, the different methodology utilised in the two surveys may account for some of the difference. The national smoking survey was a household interview survey, which could lead to substantial under-reporting by high school students as seen in studies in the US. ${ }^{4}$

Table 7 compares adolescent smoking in China and the US. ${ }^{5}$ Smoking is as prevalent among Chinese male teenagers as among their American counterparts, particularly in the older age groups. However, Chinese female teenagers are still much less likely to smoke than American female teenagers. While the trends in adolescent smoking in China are unknown, ongoing surveillance is essential.

Our study shows that smoking initiation could occur as young as five years of age. This suggests that anti-smoking efforts should be extended to the early childhood years. Parents should also become actively responsible in preventing their children from smoking.

One major characteristic in the smoking patterns among senior high school students in Beijing is that this behaviour is much more prevalent among males than females. Similar

Table 7 Smoking prevalence among Chinese and US teenagers

\begin{tabular}{llrc}
\hline & Age & Male & Female \\
\hline Beijing, China, 1988 & $14-16$ & 6.0 & 0.8 \\
(Smoking daily or weekly) & 17 & 12.2 & 1.0 \\
& 18 & 25.0 & 1.9 \\
& 19 & 26.1 & 2.3 \\
Nationwide, US, 1989 & 15 & 11.9 & 11.3 \\
(Smoking during $_{\text {preceding week) }}^{16}$ & 13.2 & 14.1 \\
& 17 & 18.2 & 17.5 \\
& 18 & 29.1 & 21.3 \\
\hline
\end{tabular}

results have been observed among Chinese junior high school students, ${ }^{9}$ and adults, ${ }^{1,3}$ as well as in the more industrialised Asian countries such as Japan ${ }^{16,17}$ and Singapore. ${ }^{18,19}$ In western countries, smoking was also practiced predominantly by men during the first half of the 20th century. ${ }^{20-22}$ However, after World War II, smoking rates among women began to rise with the gradual disappearance of the social restrictions that previously limited smoking by women., ${ }^{40-22}$ In the US, for example, $32 \%$ of women were smokers in 1965 , when the smoking rate among women reached its highest level. ${ }^{4}$ Our data show that "current fashion" was one of the most frequently cited reasons for smoking among females in both junior ${ }^{9}$ and senior high schools. Therefore, it is highly possible that female smoking prevalence in China may rise in the future. Efforts should be made to monitor smoking prevalence among females and to prevent it from increasing.

Risk factors for smoking have been studied throughout western countries. The 1989 US Surgeon General's report summarised these factors into three categories: personal characteristics, peer and family influences, and cigarette marketing. ${ }^{4}$ Our study suggests that personal characteristics and peer and family influences play similar roles in the development of smoking behaviour among Chinese students. Multiple logistic regression analysis shows that peer pressure, negative belief about smoking and health, parents' poor attitude toward smoking, and not planning higher education after graduation are strong correlates of smoking. Similar results have been found among junior high school students in our previous report. ${ }^{9}$

Studies in the US show that restricting minors' access to tobacco products may delay or prevent their decision to initiate tobacco use. ${ }^{4,23}$ Our study supports these findings by showing a dose-response relationship between parents and their children: the more often the parents ask their child to buy cigarettes, the more likely the child is a smoker. In China, although there is a law which says that "elementary and high school students will be prohibited from smoking", it has never been strictly enforced. Efforts are needed to enforce the existing law; in addition, we recommend a law that restricts minors' access to tobacco products.

It is perplexing to note that smoking among children whose parents were ex-smokers was as prevalent as among those whose parents were frequent current smokers. Similar results were also observed among junior high school students (see table 3 of our previous report ${ }^{9}$ ). A careful examination shows that this relationship cannot be explained by the confounding effects of other variables. One possible explanation for this is that parents' influence might be more important in the early adolescent period. Another possibility is that many of these students came from families where parents quit smoking for reasons other than health concerns. More studies are required to explain this unexpected finding. 


\section{Conclusions}

Among senior high school students in Beijing, $18.8 \%$ of males and $1.5 \%$ of females were found to smoke every day or every week; an additional $27.2 \%$ of males and $4.0 \%$ of females smoked occasionally. Smoking prevalence among males is comparable to that of US teenagers. Smoking among Chinese students appears to be motivated by a combination of familial, psychological, and socio-school factors, with peer group pressure being the strongest influence.

This study was supported financially by the Office of the National Committee of Patriotic Health Campaign of the People's Republic of China. The Beijing Education Bureau and the education the education departments of the districts and counties provided administrative support. Special thanks to Dr Minzhang Chen, Minister of Public Health of the People's Republic
of China, for his support; to Gary Giovino, Dana Shelton, Seth Emont, Monina Klevens, and John Peddicord, Office on Smoking and Health; James Mendlein, Office of Surveillance and Analysis, National Center for Chronic Disease Prevention and Health Promotion, US Centers for Disease Control and Prevention, for their assistance, comments and suggestions; to our colleagues in the US National Cancer Institute for their kind assistance; and to Suong Nguyen, Graduate School of Public Health, San Diego State University, for her con and editing expertise.

1 Weng XZ, Hong ZH, Chen DY. Smoking prevalence in Chinese aged 15 and above. Chin Med $\mathscr{f} 1987 ; 100(1)$ 886-92.

2 Chapman S, Leng WW. Tobacco control in the Third World. $A$ resource atlas. Penang, Malaysia: International Organization of Consumers Unions, 1990.

3 Central Committee of Patriotic Health Campaign and Ministry of Public Health of the People's Republic of China. Data compilation from the 1984 national smokin sampling survey. People's Health Press, 1988. (In Chinese)

4 US Department of Health and Human Services. Reducing report of the Surgeon General, 1989. Atlanta, Georgia: Centers for Disease Control, Office on Smoking Health 1989 (DHHS Publication No (CDC) 89-8411.)

5 US Centers for Disease Control. Cigarette smoking among youth-United States, 1989. MMWR 1991; 40(41): youth

6 US Department of Health and Human Services. The health consequences of smoking: cancer. A report of the Surgeon General, 1982. US Department of Health and Human the health consequences of smoking : 25 years of progress. A

Services, Public Health Service, Office on Smoking Health, 1982. (DHHS Publication No (PHS) 82-50179.)

7 US Department of Health and Human Services. The health consequences of smoking : cardiovascular disease. A report of the Surgeon General, 1983. US Department of Health and Human Services, Public Health Service, Office on Smoking Health, 1983 (DHHS Publication No (PHS) 84-50204.)

8 US Department of Health and Human Services. The health consequences of smoking: chronic obstructive lung disease. A report of the Surgeon General, 1984. US Department of Health and Human Services, Public Health Service, Office on Smoking and Health, 1984. (DHHS Publication No (PHS) 84-50205.)

9 Zhu BP, Liu M, Wang SQ, et al. Cigarette smoking among junior high school students in Beijing, China, 1988. Int $\mathscr{f}$ Epidemiol 1992; 21: 854-61.

10 Snedecor GW, William GC, eds. Statistical methods. (7th edn). Iowa State University Press, 1980, pp 441-53.

11 Dixon WJ, Brown MB, Engelman L, Jennrich RI. BMDP statistical software manual. University of California Press, 1990

$12 S A S / S T A T$ user's guide: Volume 1 (version 6 edn). Cary, NC: SAS Institute Inc., USA, 1990.

13 Jolliffe IT. Principal component analysis. London: SpringerVerlag, 1986

14 Hosmer DW, Lemeshow S. Applied logistic regression. New York: Wiley 1990

15 Pirie PL, Murray DM, Luepker RV. Smoking prevalence in a cohort of adolescents, including absentees, dropouts, and transfers. Am $\mathcal{F}$ Public Health 1988; 78 $(2): 176-8$.

16 Shibata A, Fukuda K, Hirohata T. Smoking habits among senior high school students and related factors. Kurume Med f 1990; 37(3): 129-40.

17 Ogawa H, Tominaga S, Gellert G, Aoki K. Smoking among junior high school students in Nagoya, Japan. Int $\mathcal{f}$ Epidemiol 1988; 17: 814-20.

18 Emmanuel SC, Ho CK, Chen AJ. Cigarette smoking among school children in Singapore. Part I Smoking Prevalence. Singapore Med f 1990; 31(3): 211-6.

19 Emmanuel SC, Ho CK, Chen AJ. Cigarette smoking among school children in Singapore. Part II Development of the cigarette habit. Singapore Med $\mathcal{f} 1991$; 32(3): 146-50.

20 Fiore MC, Novotny TE, Pierce JP, Hatziandreu EJ, Patel KM, Davis RM. Trends in cigarette smoking in the United States. The changing influence of gender and United States. The changing influe

21 US Department of Health and Human Services. The health consequences of smoking for women. A report of the Surgeon General, 1980. US Department of Health and Human Services, Public Health Service, Office of the Assistant Secretary for Health, Office on Smoking and Health, 1980 .

22 Hammond EC, Garfinkel L. Smoking habits of men and women. 7 Natl Cancer Inst 1962; 27 : 419-42.

23 US Department of Health and Human Services. Healthy People 2000: national health promotion and disease prevention objectives-full report, with commentary. Washington, DC: US Department of Health and Human Services, Public Health Service, 1991. (DHHS Publication No (PHS) 91-50212.)

\section{Appendix: Questionnaire for junior and senior high school students in the 1988 Beijing Students Smoking Survey}

Name of your school:

Are you a junior or senior high school student? (please circle one) funior Senior

Section 1: $\quad$ Social-demographic items about you.

(1) Your grade:

(2) Your gender (please circle one): Male Female

(3) Your age (based on the lunar calendar): years old

(4) Your date of birth (based on the lunar calendar): ___ year ___ month ___ate

Section 2: Questions about your family, teachers, friends, and you. Please fill in the blank with the index number of your choice. Choose the one that you think is the most appropriate.

What is your father's main occupation? (main source of income)
1. Scientist
2. Teacher
3. Medical Doctor
4. Worker
5. Government official
6. Farmer
7. Other

What is your mother's main occupation? (main source of income)
1. Scientist
2. Teache
3. Medical Doctor
4. Worker

5. Government official

6. Farmer

7. Other

What is your father's highest level of education?

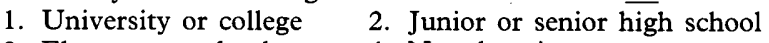

3. Elementary school 4. No education

What is your mother's highest level of education?

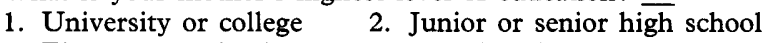

3. Elementary school 4. No education

Which of the following best describes your father's smoking status?

1. He smokes every day

2. He smokes every week

3. He smokes occasionally

4. He used to smoke daily or weekly, but has not been smoking for at least a month

5. I never saw him smoking 
(10) Which of the following best describes your mother's smoking status?

$\begin{array}{ll}\text { 1. She smokes every day } & 2 \text {. She smokes every week }\end{array}$

3. She smokes occasionally

4. She used to smoke daily or weekly, but has not been smoking for at least a month

5. I never saw her smoking

(11) Please rate your parents' attitude towards smoking.
1. Strongly against
2. Against
3. Not against

4. I don't know

Do you have sibling(s) who smoke?

0 . No one smokes 1. At least one of them smokes

Do your parents ask you to buy cigarettes for them?
0 . Never
1. Occasionally
2. Often

(14) Which of the following best describes your motive for attending school?

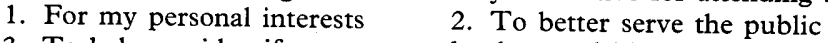

3. To help me identify my personal values and ideas

4. For the sake of my parents 5. For a diploma

6. Other

(15) Which of the following best describes your goals while attending school?
1. Achieve all-around success
2. Achieve good scores
3. Enjoy life
4. Be able to graduate and get a diploma
5. Other

(16) Which of the following best describes your goals after graduation (if possible)?

1. Pursue higher education 2. Find a good job

3. Make money 4. Other

(17) Which of the following best describes your attitude toward life?
1. Optimistic
2. Pessimistic
3. In-between

(18) Which of the following best describes your attitude toward respecting discipline?

$\begin{array}{ll}\text { 1. I am willing to respect discipline } & 2 \text {. I follow other people's suit }\end{array}$

3. I am not willing to respect discipline

(19) Which of the following best describes your beliefs about the relationship between smoking and health?

1. I believe smoking is harmful to health

2. I don't believe smoking is harmful to health 3. I have no idea

(20) Which of the following best describes your current school performance?
1. A-level
2. B-level
3. C-level
(21) To the best of your knowledge, do any of your current teachers smoke?

0 . No 1. At least one

(22) To the best of your knowledge, do any of your close friends smoke?

0 . No 1. At least one

Section 3: Questions about your smoking behaviour.

(23) Which of the following best describes your smoking status?

1. I smoke every day or almost every day

2. I smoke every week

3. I smoke occasionally (less often than once a week, on special occasions, or only puffed a few times)

4. I never smoked a cigarette

(24) At what age did you first try a cigarette?
1. $<5$ years old
2. 5-9 years old
3. 10-14 years old
4. $\geqslant 15$ years old

5. I never smoked a cigarette

(25) Which of the following best describes your primary reason for first trying a cigarette ?

$\begin{array}{lll}\text { 1. Believed smoking makes people look elegant } & 2 \text {. To imitate the behaviour of others }\end{array}$

3. To see what it was like

5. Encouraged by a friend

4. To dispel melancholy

7. To seek stimulation

6. To refresh myself

9. I never smoked a cigarette

8. Other

Questions 26-30 are to be answered by those who smoke every day or every week.

On average, how many cigarettes do you smoke per day?

$\begin{array}{lll}\text { 1. }<5 & \text { 2. 5-9 } & 3 . \geqslant 10\end{array}$

(27) When you smoke, do you usually inhale or not?

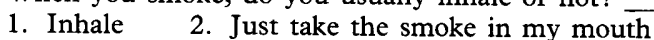

3. Both, can't say which is more often

(28) Which of the following best describes your primary reason for smoking now?

$\begin{array}{ll}\text { 1. Believe smoking makes people look elegant } & 2 \text {. To be fashionable }\end{array}$

$\begin{array}{ll}3 \text {. To dispel melancholy } & 4 \text {. To seek stimulation }\end{array}$

5. To adapt to the custom

6. To refresh myself

7. Other

(29) Where do you smoke most often?
1. My home
2. On the way to / from school
3. School
4. Friend's home
5. Other

(30) Whom do you smoke with most often?
1. I usually smoke alone
2. Friends or schoolmates
3. Family members
4. Other

(Translated by Dr Bao-Ping Zhu. Questions that were in the original questionnaire but are not reported in this paper have been excluded. The Chinese version of the questionnaire is available upon request.) 